Palavras chave:

Produtividade

polyblen

parcelamento da fertilização adubação de cobertura lixiviação

Histórico:

Recebido 22/07/2014

Aceito 10/08/2015

Keyworks:

Productivity

polyblen

split fertilization

topdressing fertilization

leaching

Correspondence: paulohenrique@ipef.br

DOI:
Paulo Henrique Muller da Silva'; Fabio Poggiani²; Ariane Andre Silva²; Ithamar Prada Neto ${ }^{3}$; Rinaldo Cesar de Paula ${ }^{4}$

\section{MORTALIDADE, CRESCIMENTO E SOLUÇÃO DO SOLO EM EUCALIPTO COM APLICAÇÃO DE FERTILIZANTE DE LIBERAÇÃO LENTA}

RESUMO: A fertilização é uma das maneiras para aumentar a produtividade e a utilização de fertilizantes de liberação lenta pode ser vantajosa, permitindo a assimilação dos nutrientes à medida que as plantas crescem. $\mathrm{O}$ objetivo deste trabalho foi avaliar o efeito da utilização de fertilizante de liberação lenta no crescimento inicial de um plantio de Eucalyptus urophylla $\times$ E.grandis, visando reduzir o número de fertilizações de cobertura. $O$ delineamento experimental foi o de blocos ao acaso, com quatro tratamentos fornecendo as mesmas quantidades de nutrientes, porém variando o parcelamento e a fonte do fertilizante: TI-Fertilizante convencional com aplicação parcelada; T2-Fertilizante convencional com aplicação em dose única aos três meses; T3-Fertilizante de liberação lenta no plantio; e T4-Fertilizante de liberação lenta aos três meses. Foram avaliadas as seguintes características: mortalidade, altura, DAP, volume de madeira, teores foliares dos macronutrientes e de $\mathrm{N}$ e $\mathrm{K}$ na solução do solo. A mortalidade média foi de $4 \%$, altura de $8,5 \mathrm{~m}$, DAP de $7,5 \mathrm{~cm}$ e volume de $24 \mathrm{~m}^{3} \cdot \mathrm{ha}^{-1}$, aos 18 meses de idade, não diferindo entre os tratamentos. Apenas o teor foliar de $\mathrm{K}$ diferiu entre os tratamentos, sendo a menor concentração com a aplicação parcelada do fertilizante. A aplicação do fertilizante de liberação lenta aos três meses proporcionou os menores teores na solução do solo. A aplicação parcelada dos nutrientes não resultou em ganho de produtividade ou benefícios nutricionais. $O$ uso de fertilizantes de liberação lenta possibilita a redução do número de fertilizações de cobertura, sem o risco de lixiviação dos nutrientes.

\section{MORTALITY, INITIAL GROWTH AND SOIL SOLUTION IN EUCALYPTS STANDS WITH SLOW RELEASE FERTILIZATION}

ABSTRACT: Fertilization is one of the most effective ways to increase crop productivity, and the use of slow release fertilizers could be advantageous, allowing the assimilation of nutrients as the plants grow. The objective was evaluating the effect of slow release fertilizers in a Eucalyptus urophylla $\times E$. grandis stand in order to reduce the number of fertilization applications. The experimental design was randomized blocks with four treatments. Treatments were applied with the same amounts of nutrients: TI -Convetional fertilizer with split application; T2-Conventional fertilizer in a single dose at 3 months; T3-Controlled-release fertilizer applied at planting; and T4-Controlled-release fertilizer applied 3 months after planting. We evaluated the mortality, initial growth, leaf nutrition and $\mathrm{N}$ and $\mathrm{K}$ in the soil solution. The experiment showed an average mortality of $4 \%$, height of $8.5 \mathrm{~m}, \mathrm{DBH}$ of $7.5 \mathrm{~cm}$ and volume of $24 \mathrm{~m}^{3} \cdot \mathrm{ha}^{-1}$ at 18 months of age, with no difference among treatments for these characteristics. There were no differences in foliar concentrations of $\mathrm{N}, \mathrm{P}, \mathrm{Ca}, \mathrm{Mg}$ and $\mathrm{S}$, only the $\mathrm{K}$ differed among treatments, with the lowest concentration at conventional fertilizer split application treatment. The application of slow release fertilizer at 3 months showed the lowest concentrations of $\mathrm{N}$ and $\mathrm{K}$ in the soil solution. The split application of nutrients showed no improvement in eucalypt growth or nutritional benefits. The use of slow-release fertilizer is possible to reduce the number of fertilization application with no risk of nutrients leaching.

I IPEF, Piracicaba, São Paulo, Brasil

2 ESALQ, Piracicaba, São Paulo, Brasil

${ }^{3}$ Produquímica Ind. e Com. S/A, São Paulo, São Paulo, Brasil

${ }^{4}$ UNESP, Jaboticabal, São Paulo, Brasil 


\section{INTRODUÇÃO}

A fertilização mineral é uma das ferramentas mais comum e efetiva que se dispõe para melhorar a qualidade química do solo e, consequentemente, aumentar a produtividade das culturas. As espécies do gênero Eucalyptus, em geral, aumentam a produtividade com a fertilização (VALE et al., 2000; SILVA et al., 2008), pois na maioria das áreas utilizadas pelo setor florestal o estoque de nutrientes no solo não é capaz de fornecer todos os elementos para um crescimento adequado (LACLAU et al., 2003). O modo e a quantidade de fertilizantes aplicados dependem das necessidades nutricionais, da fertilidade do solo, da reação dos fertilizantes com o solo, da eficiência dos fertilizantes e das condições climáticas.

O manejo nutricional de um povoamento florestal é realizado visando obter boa produtividade com o menor custo possível, mas também requer atenção na quantificação dos fluxos de nutrientes no ecossistema (LACLAU et al., 2005; SILVA et al., 2009). Em florestas plantadas, a quantidade de nutrientes exportada durante a exploração e a lixiviada são importantes na definição do balanço de nutrientes e na necessidade de aplicação de fertilizantes (MELO et al., 1995; SILVA et al., 20 I3).

Os fertilizantes de liberação lenta permitem a disponibilização gradual dos nutrientes à planta em função da temperatura e umidade do solo, e do período de crescimento das plantas (TOMASZEWSKA et al., 2002). A liberação lenta e contínua dos nutrientes é vantajosa para o eucalipto, pois permite assimilação dos nutrientes durante o crescimento do sistema radicular (POGGIANI et al., 2000).

O uso de fertilizantes de liberação lenta pode apresentar vantagens (SHARMA 1979), como: redução da mão-de-obra devido ao menor número de aplicações em cobertura; redução das taxas de queima de folhas proveniente de fertilizantes aplicados na superfície; redução da perda por volatilização; redução da perda de nutrientes via lixiviação e escoamento superficial e redução dos danos nas plantas pela salinidade. Por outro lado, existem riscos na utilização de fertilizantes de liberação controlada: a liberação pode ser lenta e causar perdas de produtividade ou a liberação pode ser rápida e ocasionar mortalidade das mudas ou mesmo a perda de nutrientes.

Os riscos de perda de nutrientes por lixiviação em plantio de eucalipto ocorrem apenas na fase inicial, principalmente durante 0 primeiro ano de estabelecimento quando ocorre maior percolação (SILVA et al., 2014), mas após a fase inicial de crescimento o risco de lixiviação é praticamente nulo (LACLAU et al., 20I3). Contudo, na área florestal a literatura é escassa em trabalhos, em condições de campo, com a aplicação de fertilizante de liberação lenta. $O$ objetivo deste trabalho foi avaliar o efeito da aplicação de fertilizante de liberação lenta no crescimento inicial de um hibrido de Eucalyptus urophylla $\times$ E. grandis e na solução do solo, visando reduzir o número de fertilizações de cobertura.

\section{MATERIAL E MÉTODOS}

\section{Local}

A área de estudo localiza-se na Fazenda Morro do Ouro, de propriedade da Duratex, município de Botucatu-SP, situada a $22^{\circ} 53^{\prime}$ de latitude sul e $48^{\circ} 26^{\prime}$ de longitude oeste, com altitude de 750 metros. A temperatura média anual é de $20,2{ }^{\circ} \mathrm{C}$, precipitação média anual de $1302 \mathrm{~mm}$, concentrada de outubro a março e precipitações médias mensais inferiores a 50 $\mathrm{mm}$ nos meses mais secos (Figura I). O clima é o Cwa de acordo com a classificação de Koeppen, e o solo, um Latossolo Vermelho Amarelo álico com textura média, cuja caracterização química encontra-se na Tabela I.

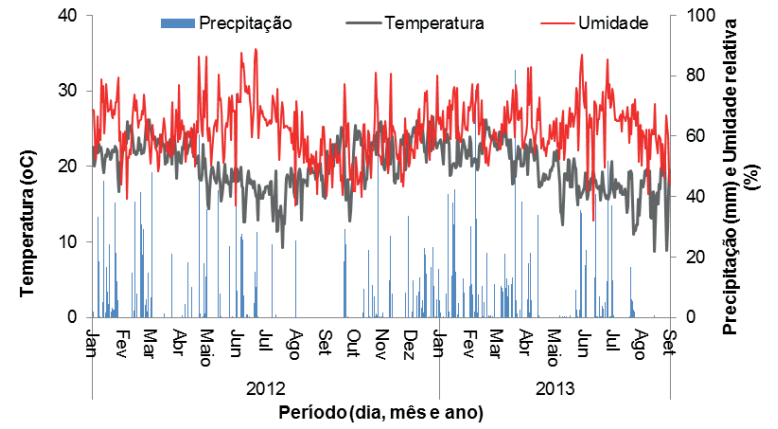

FIGURA 1 Precipitação, temperatura e umidade do ar diária durante o experimento.

FIGURE 1 Dialy rainfall, temperature e air moisture during the experiment time frame.

\section{Implantação e descrição do experimento}

O experimento foi instalado em janeiro de 2012, com o plantio de mudas clonais de um híbrido de Eucalyptus urophylla $\times$ E. grandis. O preparo do 
TABELA 1 Caracterização química inicial do solo da área experimental.

TABLE 1 Initial soil characterization at the experimental area.

\begin{tabular}{|c|c|c|c|c|c|c|c|c|c|c|c|c|}
\hline \multirow{2}{*}{$\begin{array}{l}\text { Prof. } \\
(\mathrm{cm})\end{array}$} & \multirow{2}{*}{$\begin{array}{c}\mathrm{pH} \\
\mathrm{CaCl}_{2}\end{array}$} & \multirow{2}{*}{$\begin{array}{c}\text { M.O. } \\
(\text { g.dm } \\
\text { (-3) }\end{array}$} & \multirow{2}{*}{$\begin{array}{c}\text { P* } \\
\left(\mathrm{mg} \cdot \mathrm{dm}^{-3}\right)\end{array}$} & $\mathbf{K}$ & $\mathbf{C a}$ & Mg & $\mathbf{H}+\mathbf{A l}$ & Al & SB & \multirow{2}{*}{ СTC } & \multirow{2}{*}{$\begin{array}{c}\mathrm{V} \\
(\%)\end{array}$} & \multirow{2}{*}{$\begin{array}{c}\mathrm{m} \\
(\%)\end{array}$} \\
\hline & & & & \multicolumn{6}{|c|}{------------- mmolc dm-3 -------------- } & & & \\
\hline $0-20$ & 4 & 19 & 7 & 0,5 & 3 & 3 & 52 & II & 7 & 59 & II & 63 \\
\hline $20-40$ & 4 & 9 & 4 & 0,3 & 2 & 2 & 38 & 8 & 4 & 42 & 10 & 65 \\
\hline
\end{tabular}

* extração resina Prof. - profundidade de coleta das amostras de solo; p.H. - potencial hidrogeniônico; M.O. matéria orgânica; $\mathrm{P}, \mathrm{K}, \mathrm{Ca}, \mathrm{Mg}, \mathrm{H}+\mathrm{Al}, \mathrm{Al}$ - respectivamente, teores de fósforo, potássio, cálcio, magnésio, hidrogênio + alumínio, alumínio; SB - soma de bases; CTC - capacidade de troca catiônica; $\mathrm{V}$ - saturação por bases; $\mathrm{m}$ - saturação em alumínio.

solo consistiu da subsolagem da linha de plantio num espaçamento de $3 \times 2 \mathrm{~m}$, sem a aplicação de fertilizantes, a qual foi realizada manualmente com recipientes calibrados simulando a aplicação comercial após o preparo de solo e demarcação das parcelas. $O$ delineamento experimental foi blocos ao acaso, com quatro tratamentos e seis repetições. Cada parcela experimental foi composta por 96 plantas, distribuídas em oito linhas de 12 plantas, com bordadura dupla, totalizando uma área de $576 \mathrm{~m}^{2}$ por parcela, tendo a parcela útil 32 plantas e área de $192 \mathrm{~m}^{2}$.

Foram estudados quatro tratamentos fornecendo as mesmas quantidades de nutrientes, variando o parcelamento e a fonte do fertilizante: TIFertilizante convencional com aplicação parcelada: Comercial-Parcelada; T2-Fertilizante convencional com aplicação em dose única aos três meses: Comercial - 3 meses; T3-Fertilizante de liberação lenta no plantio: Polyblen - Plantio; e T4-Fertilizante de liberação lenta aos três meses: Polyblen - 3 meses (Tabela 2).

\section{Avaliação da mortalidade e crescimento inicial}

Foram realizadas avaliações de mortalidade e altura aos 6, 12 e 18 meses após o plantio e do diâmetro a altura do peito (DAP, a I,30 m do solo) aos 18 meses. A partir da altura e do DAP, obteve-se o volume de madeira com casca (equação I) conforme utilizada pela empresa Duratex, sendo: Vol - volume $\mathrm{m}^{3} \cdot \mathrm{pl}^{-1}$; D - DAP em $\mathrm{cm}$ e $\mathrm{H}$ - altura em $\mathrm{m}$.

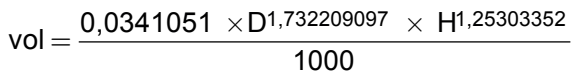

TABELA 2 Tratamento de fertilização de plantio e de cobertura aplicados.

TABLE 2 Planting and topdressing fertilization treatments applied.

\begin{tabular}{|c|c|c|c|c|c|c|c|c|c|c|c|}
\hline \multirow{3}{*}{ Tratamentos } & \multirow{2}{*}{\multicolumn{2}{|c|}{$\begin{array}{l}\text { Plantio } \\
\left(\mathrm{g} \cdot \mathrm{pl}^{-1}\right)\end{array}$}} & \multicolumn{6}{|c|}{ Cobertura (g.pl- $\left.{ }^{-1}\right)$} & \multirow{2}{*}{\multicolumn{3}{|c|}{$\begin{array}{c}\text { Nutrientes } \\
\left(\mathbf{k g}^{-h^{-1}}\right)^{-1}\end{array}$}} \\
\hline & & & \multicolumn{2}{|c|}{3 meses } & \multicolumn{2}{|c|}{10 meses } & \multicolumn{2}{|c|}{18 meses } & & & \\
\hline & Dose & N-P-K & Dose & N-P-K & Dose & N-P-K & Dose & N-P-K & $\mathbf{N}$ & $\mathbf{P}_{2} \mathbf{O}_{5}$ & $\mathrm{~K}_{2} \mathrm{O}$ \\
\hline $\begin{array}{l}\text { I -Comercial - } \\
\text { Parcelada }\end{array}$ & 162 & $06-30-10$ & 138 & $13-00-15$ & 168 & II-00-24 & 132 & $00-00-57$ & 77 & 81 & 254 \\
\hline \multirow[t]{3}{*}{$\begin{array}{l}\text { 2- Comercial - } \\
3 \text { meses }\end{array}$} & 162 & $06-30-10$ & 138 & $13-00-15$ & & & & & 77 & 81 & 254 \\
\hline & & & 168 & II-00-24 & & & & & & & \\
\hline & & & 132 & $00-00-57$ & & & & & & & \\
\hline \multirow[t]{2}{*}{$\begin{array}{c}\text { 3-Polyblen* - } \\
\text { Plantio }\end{array}$} & 162 & $06-30-10$ & & & & & & & 77 & 81 & 254 \\
\hline & 395 & Polyblen & & & & & & & & & \\
\hline $\begin{array}{l}\text { 4- Polyblen - } 3 \\
\text { meses }\end{array}$ & 162 & $06-30-10$ & 395 & Polyblen & & & & & 77 & 81 & 254 \\
\hline
\end{tabular}

*Polyblen: fertilizante de liberação lenta com nutrientes encapsulados com enxofre e polímero orgânico, a liberação dos nutrientes ocorre até 6 meses após a aplicação. 


\section{Concentração dos nutrientes nas folhas}

Aos 12 meses após o plantio foram coletadas folhas para análise de macronutrientes. Foram retiradas quatro folhas dos diferentes pontos cardiais por planta das nove plantas centrais de cada parcela para constituírem uma amostra composta por repetição. As amostras foram acondicionadas em sacos de papel e submetidas à secagem em estufa com circulação forçada de ar, regulada a $65^{\circ} \mathrm{C}$. Em seguida foram moídas em moinho tipo Willey com peneira de 40 mesh, para realização das análises químicas, conforme métodos descritos por Malavolta et al. (1997).

\section{Avaliação da concentração de $\mathrm{Ne} \mathrm{K}$ na solução do solo}

Para avaliar a solução do solo foram instalados três extratores de solução em quatro repetições de cada tratamento, nas profundidades de 20 e $100 \mathrm{~cm}$. A sucção foi realizada com uma bomba manual e as coletas da solução foram realizadas, $48 \mathrm{~h}$ após a sucção, durante o primeiro ano, sempre que a umidade do solo permitisse a coleta $(U$ $>12 \%$ ). As amostras foram coletadas mensalmente entre fevereiro e julho de 2012, sendo realizada mais três vezes entre dezembro de 2012 e março de 20I3. Avaliou-se a concentração de $\mathrm{N}$ e K na solução do solo, com o uso dos aparelhos portáteis Horiba compact $\mathrm{NO}_{3}$ - meter B-343 e Horiba compact íon meter C-I3I K+.

\section{Análises estatísticas}

Para verificar a existência de diferenças significativas entre os tratamentos foram feitas análises de variância (teste
F) para cada característica e idade avaliada. Para os dados em que foram observadas diferenças significativas, foi aplicado o teste complementar de Tukey, a $5 \%$ de probabilidade (PIMENTEL - GOMES e GARCIA, 2002).

\section{RESULTADOS E DISCUSSÃO}

\section{Avaliação da mortalidade e crescimento inicial}

Não foram observadas diferenças significativas na mortalidade entre os tratamentos, a qual variou de 3 a $6 \%$. Não houve diferença significativa na altura das plantas nos diferentes tratamentos de fertilização (Figura 2).

Resultado coerente com outros experimentos de fertilização de eucalipto (SILVA et al., 2008; SILVA et al., 20I3), cujo crescimento em altura não diferencia as fertilizações estudadas após o primeiro ano, sendo que a possível resposta ocorre apenas no diâmetro das plantas, refletindo no volume de madeira produzida. ○ que não foi observado neste estudo que aos 18 meses de idade, o DAP e o volume de madeira foram semelhantes entre os tratamentos, produção média de $24 \mathrm{~m}^{3} \cdot \mathrm{ha}^{-1}$ (Figura 3).

Neste experimento, foi observado que a aplicação da fertilização de cobertura em dose única ou parcelada, independentemente do fertilizante, não diferenciou o crescimento dos eucaliptos em altura, diâmetro ou volume de madeira e que foram condizentes com o esperado para a silvicultura da região (SILVA et al., 2008, 20I3; STAPE et al., 2008).

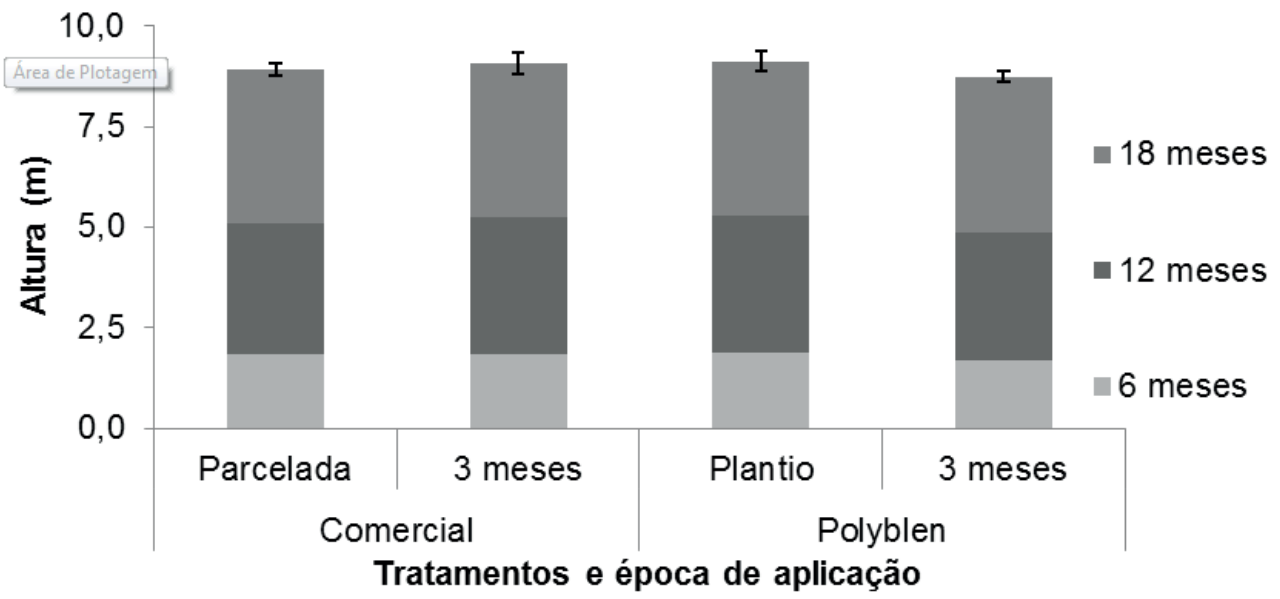

FIGURA 2 Média ( \pm desvio padrão) de altura de Eucalyptus urophylla $\times$ E. grandis submetidas a quatro tratamentos de fertilizantes, após 6, 12 e 18 meses do plantio.

FIGURE 2 Average ( \pm standard deviation) of Eucalyptus urophylla $\times$ E. grandis height with different fertilizer treatments at 6 , 12 and 18 months after planting. 

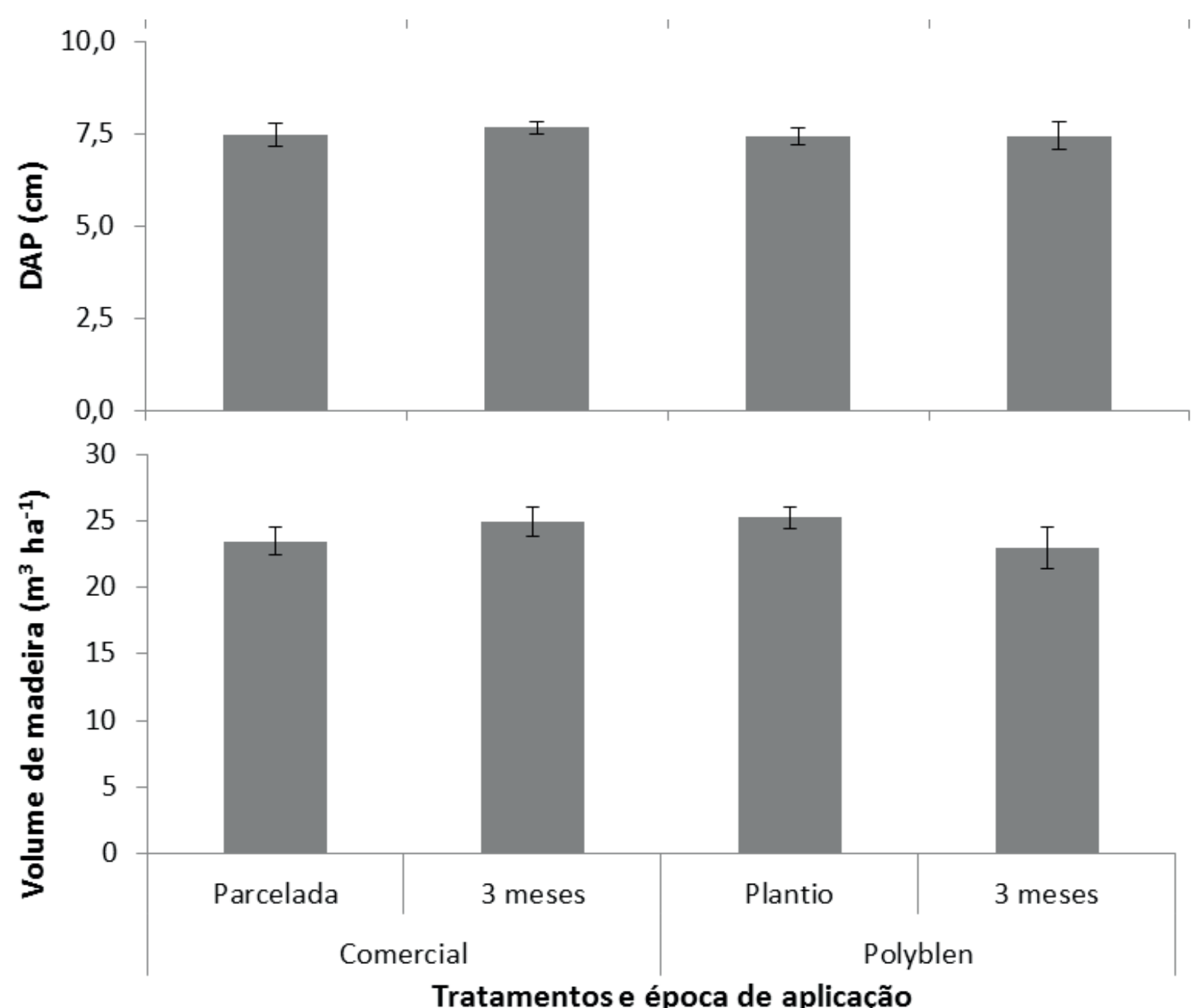

FIGURA 3 Média ( \pm desvio padrão) do DAP e do volume de madeira de Eucalyptus urophylla $x$ E. grandis nos quatro tratamentos de fertilização, 18 meses após o plantio.

FIGURE 3 Average ( \pm standard deviation) DBH and wood volume of Eucalyptus urophylla $\times$ E. grandis with four fertilization treatments, 18 months after planting.

Concentração dos nutrientes nas folhas

Não foram observadas diferenças nas concentrações foliares do N, P, Ca, Mg e S (Tabela 3) aos 12 meses após o plantio. Apenas o K diferiu entre os tratamentos, sendo que a aplicação comercial com parcelamento da fertilização de cobertura resultou na menor concentração foliar $\left(4,3 \mathrm{~g} \cdot \mathrm{kg}^{-1}\right)$. Esse menor teor de $\mathrm{K}$ se deve a menor quantidade aplicada do nutriente até ○ momento da avaliação no tratamento com aplicação parcelada. Esse nutriente poderia restringir o crescimento das plantas, mas não foi observada influencia do baixo teor do nutriente com aplicação parcelada no crescimento do eucalipto até o $18^{\circ}$ mês após o plantio.
Dell et al. (200I) determinaram faixas de concentrações "adequadas" de nutrientes para o eucalipto na fase inicial de crescimento, sendo para o $\mathrm{N}$ de 18 a 30 g. $\mathrm{kg}^{-1}$; P de I a 3 g. $\mathrm{kg}^{-1} ; \mathrm{K}$ de 6 a $18 \mathrm{~g} . \mathrm{kg}^{-1}$; Ca de 3 a 8 g.kg '; $\mathrm{Mg} I$ a $3 \mathrm{~g} \cdot \mathrm{kg}^{-1}$ e S de I,5 a $3 \mathrm{~g} \cdot \mathrm{kg}^{-1}$. Considerando essas faixas, $\circ \mathrm{N}, \mathrm{P}$ e $\circ \mathrm{Mg}$ estariam em teores adequados no período experimental. $\mathrm{O} \mathrm{K}$ encontra-se deficiente em todos os tratamentos, independentemente da forma e fonte do fertilizante. Todavia, mesmo que as concentrações de $\mathrm{K}$ nas folhas estejam abaixo da faixa mencionada pelos autores, não é possível afirmar que a produtividade esteja sendo afetada, pois as plantas estão apresentando crescimento compatível com a idade e a quantidade aplicada é adequada para a cultura, conforme discutido por Silva et al. (20l3). 
TABELA 3 Teores de macronutrientes em folhas de Eucalyptus urophylla $\times$ E. grandis submetidos a quatro tratamentos de fertilização, aos 12 meses de idade.

TABLE 3 Leaf macronutrients concentration in Eucalyptus urophylla $x$ E. grandis with four fertilization treatments, 12 months after planting.

\begin{tabular}{|c|c|c|c|c|c|c|}
\hline \multirow{2}{*}{ Tratamentos } & $\mathbf{N}$ & $\mathbf{P}$ & $\mathbf{K}^{*}$ & $\mathbf{C a}$ & Mg & $\mathbf{S}$ \\
\hline & \multicolumn{6}{|c|}{ 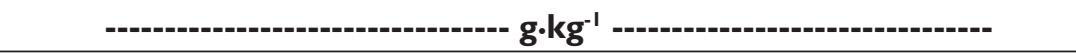 } \\
\hline I- Comercial - Parcelada & 21,5 & $\mathrm{I}, 3$ & $4,3 \mathrm{~B}$ & 2,6 & 1,4 & $\mathrm{I}, \mathrm{I}$ \\
\hline 2- Comercial - 3 meses & 21,8 & 1,3 & $5,2 \mathrm{~A}$ & 2,7 & 1,3 & 1,2 \\
\hline 3- Polyblen - Plantio & 21,1 & 1,3 & $4,9 \mathrm{~A}$ & 3,0 & 1,5 & $\mathrm{I}, \mathrm{I}$ \\
\hline 4- Polyblen - 3 meses & 19,9 & $\mathrm{I}, 4$ & $5,2 \mathrm{~A}$ & 2,6 & $\mathrm{I}, 5$ & $\mathrm{I}, \mathrm{I}$ \\
\hline
\end{tabular}

* único nutriente cujas concentrações foliares apresentaram diferenças significativas entre os tratamentos, pela análise de variância e teste complementar de Tukey $(P<0.05)$.

\section{Avaliação da concentração de $\mathrm{Ne} \mathrm{K}$ na solução do solo}

A aplicação do Polyblen no plantio (Tratamento 3) aumentou consideravelmente a concentração de $\mathrm{N}-\mathrm{NO}_{3}{ }^{-}$e de $\mathrm{K}$ na solução do solo, nas profundidades de 20 e $100 \mathrm{~cm}$ logo após o plantio, sendo que os valores de
$\mathrm{K}$ foram inferiores ao aumento ocasionado pela aplicação do fertilizante convencional em dose única aos três meses (Figuras 4 e 5). $O$ aumento na concentração dos nutrientes ocorreu logo após a aplicação dos fertilizantes, persistindo por alguns meses, sendo maior na camada mais superficial, principalmente para $\circ \mathrm{K}$, que por ser

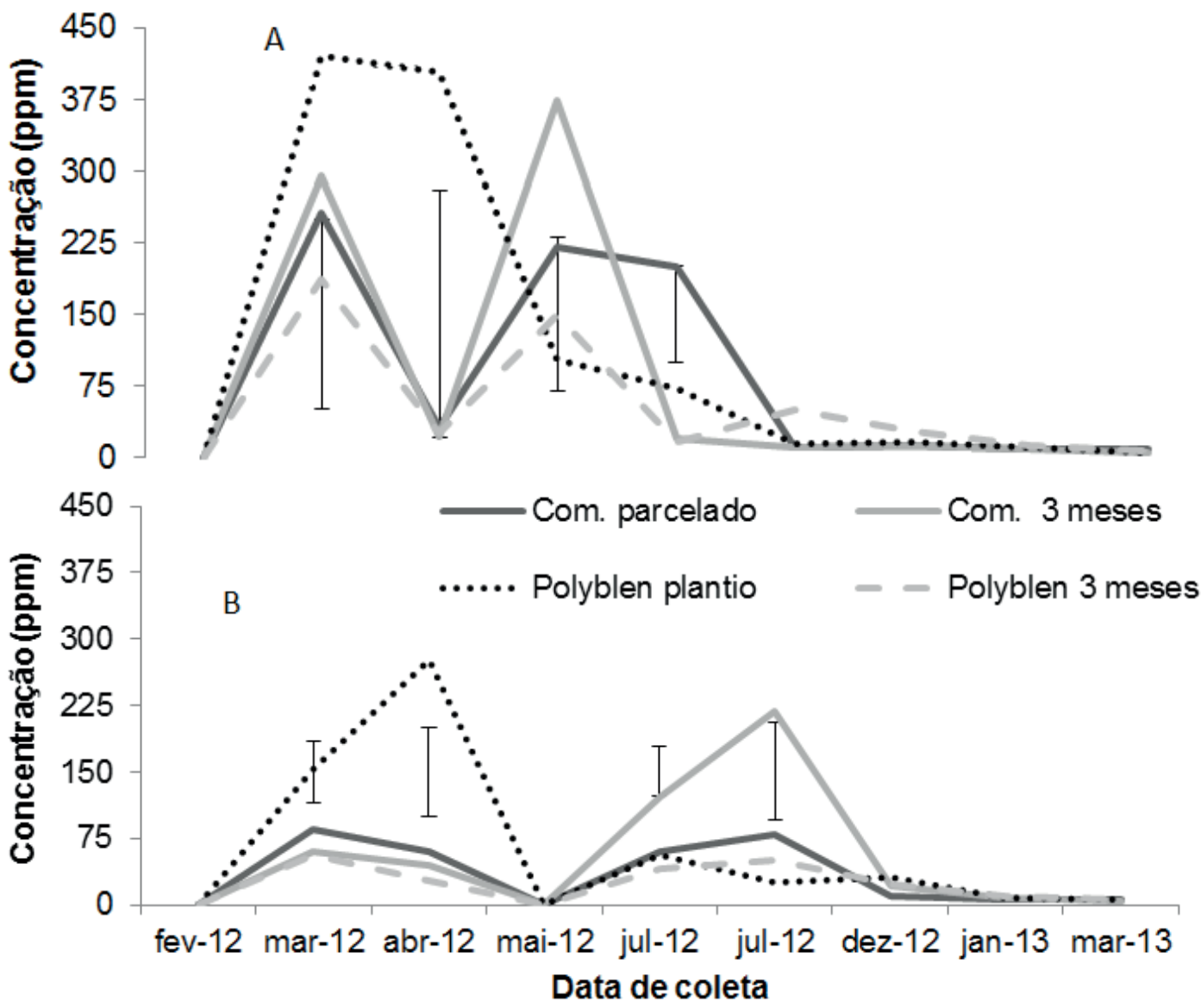

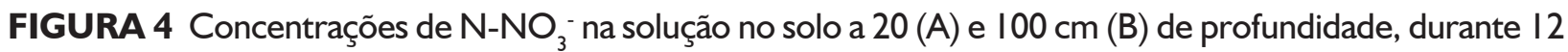
meses após plantio de Eucalyptus urophylla $\times E$. grandis (barras = diferença mínima significativa).

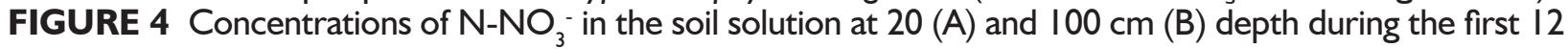
months after planting of Eucalyptus urophylla $\times E$. grandis (bars indicate least significant difference). 

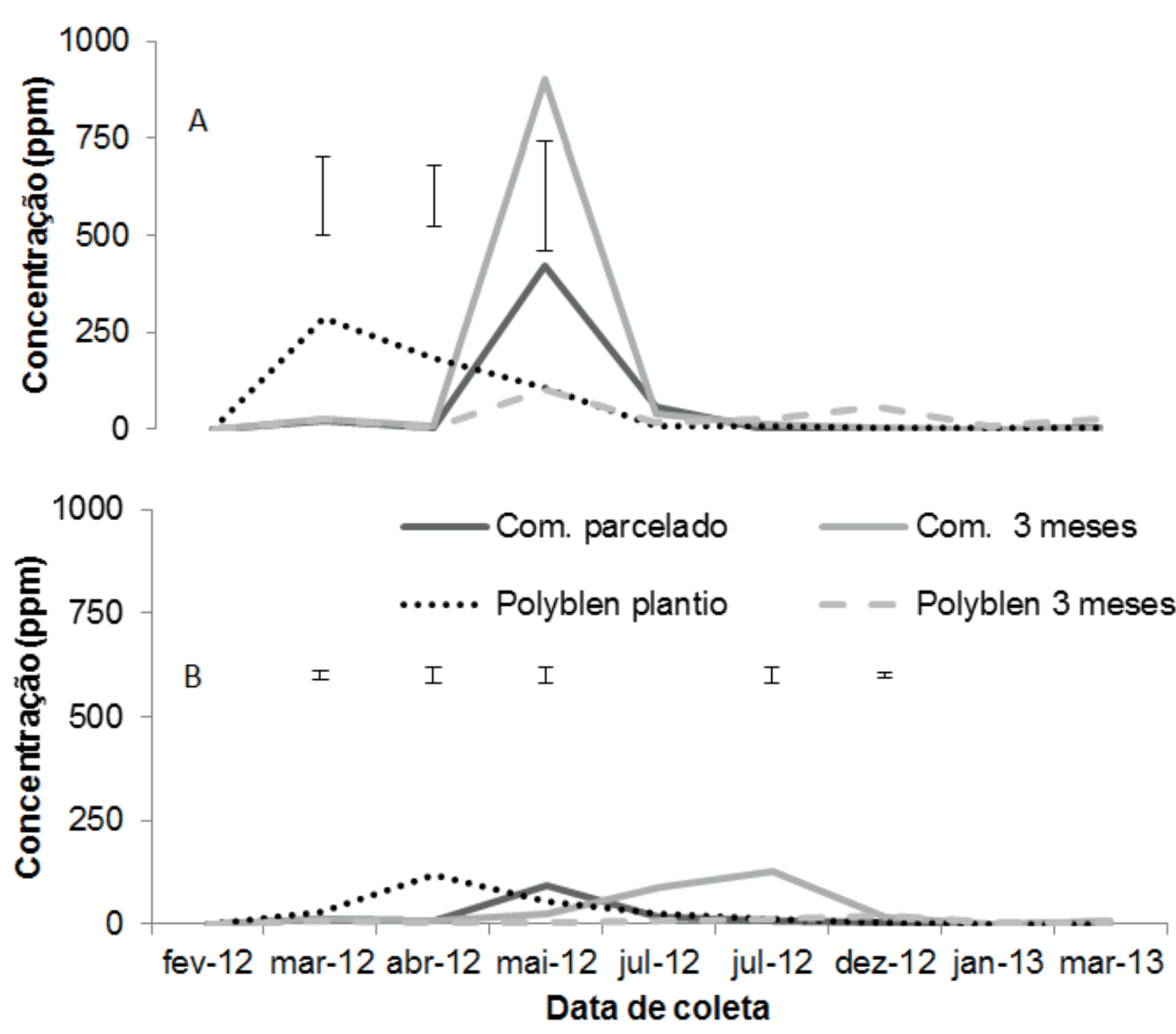

FIGURA 5 Concentrações de $K$ na solução no solo a 20 (A) e $100 \mathrm{~cm}$ (B) de profundidade, durante 12 meses após plantio de Eucalyptus urophylla $\times$ E. grandis (barras = diferença mínima significativa).

FIGURE 5 Concentrations of $K$ in the soil solution at $20(A)$ and $100 \mathrm{~cm}$ (B) depth during the first 12 months after planting of Eucalyptus urophylla $\times$ E. grandis (bars indicate least significant difference).

um cátion movimenta-se mais lentamente do que o $\mathrm{N}$ (SILVA et al., 20I3), e pela maior quantidade aplicada do elemento $\left(K=160\right.$ e $\left.N=80 \mathrm{~kg} \cdot \mathrm{ha}^{-1}\right)$.

aumento na concentração dos nutrientes na solução do solo na camada mais superficial gera maior disponibilidade para as plantas dos nutrientes aplicados. Mas o aumento rápido da concentração da solução do solo em profundidade gera o risco de lixiviação, pois pouco tempo após o plantio não existe sistema radicular em profundidade para assimilar os nutrientes. $O$ tratamento 4 (Polyblen -3 meses), em todas as análises proporcionou a menor concentração de $\mathrm{K}$ e $\mathrm{N}$, apesar de ter sido aplicada toda a fertilização de cobertura aos três meses. $\mathrm{O}$ aumento da concentração do $\mathrm{N}$ e $\mathrm{K}$ a $100 \mathrm{~cm}$ de profundidade no tratamento 4 ocorreu alguns meses após o plantio, idade em que segundo Silva et al. (20I I) o eucalipto já apresenta sistema radicular desenvolvido e com capacidade de absorver os nutrientes em profundidade.

Segundo Mitchell e Smethurst (2008), a fertilização nitrogenada além de aumentar a produtividade do eucalipto, pode aumentar a disponibilidade de bases no solo durante alguns anos, dependendo da fonte e quantidade do fertilizante. Todavia, $\circ \mathrm{N}$ e os cátions trocáveis podem ser lixiviados em profundidade, principalmente nos solos arenosos e em regiões tropicais, geralmente utilizados para a implantação de povoamentos florestais.

As concentrações de $\mathrm{N}$ e $\mathrm{K}$ na solução do solo, neste estudo, foram superiores às encontradas por 
Laclau et al. (2003) em plantios de eucalipto no Congo, mas próximas à concentração de $\mathrm{K}$ e superiores à de $\mathrm{N}$ encontradas por Silva et al. (2013) em plantios de eucalipto no estado de São Paulo. Um dos motivos da diferença em relação ao trabalho de Laclau et al. (2003) é que as quantidades de fertilizantes aplicadas no Brasil são superiores às aplicadas no Congo.

A aplicação do fertilizante de liberação lenta em cobertura em dose única aos três meses de idade (Tratamento 4) apresentou menor risco de lixiviação do $\mathrm{N}$ e $\mathrm{K}$, menor até que o tratamento com aplicação parcelada, considerando que foi realizada a aplicação em dose única de elevada quantidade desses nutrientes $\left(\mathrm{N}=67 \mathrm{~kg} \cdot \mathrm{ha}^{-1}\right.$ e $\left.\mathrm{K}_{2} \mathrm{O}=240 \mathrm{~kg} \cdot \mathrm{ha}^{-1}\right)$ e não foi observada elevação da solução do solo a um metro de profundidade. Com este método, o risco de lixiviação é praticamente nulo, considerando que o sistema radicular pode absorver os nutrientes devido ao tempo de movimentação do nutriente no solo e o ritmo de crescimento do sistema radicular do eucalipto, conforme observado em outros trabalhos em condições edafoclimáticas semelhantes às estudadas (SILVA et al., 20 I I, 20 I3; LACLAU et al., 20 I3).

Neste trabalho a aplicação parcelada dos nutrientes não gerou benefícios nutricionais ou em produtividade corroborando com os resultados obtidos por Silva et al. (20l3) que estudaram o parcelamento da fertilização em um plantio de eucalipto em solo arenoso (Neossolo Quartzarênico-RQ). A principal vantagem na redução do número de parcelas da fertilização de cobertura é a redução da mão-de-obra que é cada vez mais escassa e onerosa para implantação e manutenção florestal. Mas a aplicação de doses elevadas de fertilizantes convencionais pode gerar a mortalidade das mudas e a lixiviação dos nutrientes nos primeiros meses após o plantio, sendo que o risco de lixiviação dos nutrientes é praticamente nulo com a aplicação de fertilizantes de liberação lenta em dose única alguns meses após o plantio.

\section{CONCLUSÕES}

A aplicação parcelada de fertilizante convencional e a aplicação de fertilizante de liberação lenta em dose única não resultaram em diferenças em mortalidade, produtividade ou benefícios nutricionais às plantas de Eucalyptus urophylla $\times$ E. grandis até 18 meses de idade.

A aplicação do fertilizante de liberação lenta aos três meses possibilita a redução do número de fertilizações de cobertura, sem diminuir a produtividade e sem o risco de lixiviação dos nutrientes.

\section{AGRADECIMENTOS}

Agradecemos $\circ$ apoio das empresas Produquímica e Duratex e aos colegas Cesar Negrette, Jarbas Silva Borges, Paulo R. C. de Sousa e Raul Chaves e aos revisores pelas contribuições que foram importantes para obtenção do artigo.

\section{REFERÊNCIAS}

BENNETT, L.T.; WESTON, C.J.; JUDD, T.S.; ATTIWILL, P.M.; WHITEMAN, P.H. The effects of fertilizers on early growth and foliar nutrient concentrations of three plantation eucalypts on high quality sites in Gippsland, southeastern Australia. Forest Ecology and Management, Amsterdam, v.89, p.213-226, 1996.

DELL, B.; MALAJCZUK, D.; XU, D.; GROVE, T.S.Nutrient Disorders in Plantation Eucalypts. 2nd.ed., Camberra: ACIAR, 188p. 200I.

JUDD, T.S.; BENNETT, L.T.; WESTON, C.W.; ATTIWILL, P.M.; WHITEMAN, P.H. The response of growth and foliar nutrients in young Eucalyptus globulus (Labill.) plantations in Gippsland, southeastern Australia. Forest Ecology and Management, Amsterdam, v.82, p.87-101, 1996.

LACLAU, J-P; RANGER, J; NZILA, J D; BOUILLET, J-P; DELEPORTE, P. Nutrient cycling in a clonal stand of Eucalyptus and an adjacent savanna ecosystem in Congo: 2. Chemical composition of soil solutions. Forest Ecology and Management, Amsterdam, v. I80, p. 527-544, 2003.

LACLAU, J. P; RANGER, J; DELEPORTE, P; NOUVELLON, Y; SAINT-ANDRE, L; MARLET, S; BOUILLET, J-P. Nutrient cycling in a clonal stand of Eucalyptus and an adjacent savanna ecosystem in Congo 3. Input-output budgets and consequences for the sustainability of the plantations. Forest Ecology and Management, Amsterdam, v. 210, p.375-39I, 2005.

LACLAU J-P., DA SILVA E.A., RODRIGUES LAMBAIS G., BERNOUX M., LE MAIRE G., STAPE J.L., BOUILLET J-P., GONÇALVES J.L.M., JOURDAN C. AND NOUVELLON Y. Dynamics of soil exploration by fine roots down to a depth of $10 \mathrm{~m}$ throughout the entire rotation in Eucalyptus grandis plantations. Front. Plant Science. v.4, p.243, 2013.

MALAVOLTA, E.; VITTI, G. C.; OLIVEIRA, S. A. Avaliação do estado nutricional das plantas: princípios e aplicações. 2. ed. Piracicaba: POTAFOS, 319 p. 1997. 
MITCHELL, A.D.; SMETHURST, P.J. Base cation availability and leaching after nitrogen fertilisation of a eucalypt plantation. Australian Journal Soil Research, Camberra, v.46, p.445454, 2008.

MELO, V.F; NOVAIS, R.F.; BARROS, N.F; FONTES, M.P.F; COSTA, L.M. Balanço nutricional, eficiência de utilização e avaliação da fertilidade do solo em P, K, Ca e Mg em plantios de eucalipto no Rio Grande do Sul. IPEF, Piracicaba, v. 48/49, p.8-17, 1995.

SILVA, E.V.; BOUILLET, J. P.; GONÇALVES, J.L.M.; ABREU JUNIOR, C.H.; TRIVELIN, P.C.O.; HINSINGER, P.; JOURDAN, C., NOUVELLON, STAPE, J.L.; LACLAU, J-P. Functional specialization of Eucalyptus fine roots: contrasting potential uptake rates for nitrogen, potassium and calcium tracers at varying soil depths. Functional Ecology, London, v.25, p. 996-1006, 201 I.

SILVA, P. H. M.; POGGIANI, F;; STAPE, J. L. Crescimento de Eucalyptus grandis tratado com diferentes doses de lodos de esgoto úmido e seco, condicionados com polímeros. Scientia Forestalis, Piracicaba, v. 36, p. 79-88, 2008.

SILVA, P. H. M.; POGGIANI, F.; BRITO, J. O. et al. Produção de óleo essencial e balanço nutricional em Corymbia citriodora adubado com lodo de esgoto em diferentes espaçamentos. Cerne, Lavras, v. I5, p. 346-354, 2009.

SILVA, P.H.M.; POGGIANI, F; LIBARDI, P. L.; GONÇALVES, A. N. Fertilizer management of eucalypt plantations on sandy soil in Brazil: Initial growth and nutrient cycling. Forest Ecology and Management, Amsterdam,v.30I, p. 67-7I, 2013.
SILVA, P.H.M.; POGGIANI, F.; LIMA, W.P.; LIBARDI, P. L. Soil water dynamics and litter production on eucalypt and native vegetation in Southeastern Brazil. Scientia Agricola, Piracicaba, v.7I, n.5, p. 374-379, 2014.

SHARMA, G. C. Controlled-release fertilizers and horticultural applications. Scientia Horticulturae, v. I I, p. I07-I29, 1979.

STAPE, J.L.; BINKLEY, D.; RYAN, M.G. Production and carbon allocation in a clonal Eucalyptus plantation with water and nutrient manipulations. Forest Ecology and Management, Amsterdam, v. 255, p. 920-930, 2008.

PIMENTEL-GOMES, F; GARCIA, C.H. Estatística aplicada a experimentos agronômicos e florestais: exposição com exemplos e orientações para uso de aplicativos. Piracicaba: FEALQ, 2002, 309p.

POGGIANI, F;; GUEDES, M.C.; BENEDETTI, V. Aplicabilidade de biossólido em plantações florestais: I. reflexo no ciclo dos nutrientes. In: Bettiol, W. Camargo, O. A. (Ed). Impacto ambiental do uso agrícola do lodo de esgoto. EMBRAPA Meio Ambiente, Jaguariuna, cap.8, p. I63-178, 2000.

TOMASZEWSKA, M.; JAROSIEWICZ, A.; KARAKULSKI, K. Physical and chemical characteristics of polymer coating in CRF formulation. Science Direct, v. I46, p.319-323, 2002.

VALE, A.T.V.; BRASIL, M.A.M.; CARVALHO, C.M.; VEIGA, R.A.A. Produção de energia do fuste de Eucalyptus grandis Hill Ex-Maiden e Acacia mangium Willd em diferentes níveis de adubação. Cerne, Lavras, v.6, p.83-88, 2000. 